\title{
Spectrum Sensing in Cognitive Radio Networks: Threshold Value Optimization and Analysis
}

\author{
Kenan KOÇKAYA ${ }^{1 *}$, İbrahim DEVEL $\dot{I}^{2}$
}

\begin{abstract}
Cognitive radio is a technology developed for the effective use of radio spectrum sources. The spectrum sensing function plays a key role in the performance of cognitive radio networks. In this study, we propose an online learning algorithm for the energy detection scheme, which aims to maximizing spectrum detection performance. Optimal threshold value, which is critical for the determination of the absence or the presence of a licensed user, was mathematically expressed in accordance with the balance between probability of detection and probability of false alarm. Performance of the proposed algorithm was tested on non-fading and different fading channels for low signal-to-noise ratio (SNR) regime with noise uncertainty. In conclusion of the simulation studies, improvement in spectrum detection performance according to optimal threshold value selection was observed.
\end{abstract}

Keywords: Spectrum Detection, Energy Detection, Threshold, Machine Learning Algorithm, Online Learning Algorithm.

${ }^{*}$ Correspondence:

kkockaya@cumhuriyet.edu.tr

${ }^{1}$ Department of Divriği Nuri Demirağ Vocational High School, Sivas Cumhuriyet University in Sivas, 58300 Divriği Sivas, Turkey 


\section{Introduction}

Wireless communication systems are undergoing rapid development in order to meet the changing demands and needs of people. The increase in wireless applications and services made it essential to address spectrum scarcity problem. Measurements made by the Federal Communications Commission (FCC) of the United States telecommunications authority have shown that licensed bands are not used at a rate up to $90 \%$. The results of the measurement were published by the FCC Spectrum Policy Task Force group in the report entitled "FCC Report of the Spectrum Efficiency Working Group" [1], [2].

In recent years, a lot of research has been done on the effective use of these spectrum bands which are either empty or are not used at full capacities. One of the notable concepts in these researches is the cognitive radio concept, introduced by Mitola in 1999 [3]. Cognitive radio is a software-based technology that detects the electromagnetic environment in which it operates, detects unused frequency bands and adapts the radio working parameters to broadcast in these bands [4]. Cognitive radio systems enable the detection of unused spectrum bands, and allow secondary users to employ unused spectrum bands without the need of primary systems intervention.

Spectrum sensing is a critical issue of cognitive radio technology because of shadowing, fading, and time-varying natures of wireless channels. Some of the techniques used for spectrum sensing are matched filter detection, cyclostationary feature detection, eigenvalue detection and energy detection [5], [6]. Matched filtering detection methods with shorter detection periods are preferred if certain signal information is known, such as bandwidth, operating frequency, modulation type and grade, pulse shape and frame structure of the primary user [7], [8]. The detection performance of this method is largely depends on the channel response. To overcome this, it requires perfect timing and synchronization in both physical and medium access control layers. This situation increases the complexity of calculation [9]-[11]. Cyclostationary detection is a method for detecting primary user transmissions by exploiting the cyclostationarity features of the received signals [12], [13]. It exploits the periodicity in the received primary signal to identify the presence of primary users. In this way, the detector can distinguish primary user signals, secondary user signals or interference. However, the performance of this detection method depends on a sufficient number of samples, which increases the computational complexity. If there is no prior information about the noise signal or the primary user signal, the eigenvalue detection method is used [14], [15]. In the eigenvalue-based methods utilizes the eigenvalues of the covariance matrix of the received signal to detect the absence or the presence of the primary signal [16], [17]. Nevertheless, having a high operational complexity is a disadvantage of this method. Similarly, if the information of the primary users are not known precisely, energy detection based methods with low mathematical and hardware complexities are preferred [18], [19].

Energy detection is a spectrum sensing technique where measuring the received signal energy and deciding on the presence or absence of the primary user, by comparing the received energy level with a threshold. Threshold function calculation is based on noise power. Numerous studies have been carried out in the literature to obtain the optimal threshold value expression, and to improve spectrum sensing performance [20]-[25]. In [20], the authors proposed a new method for adaptive threshold selection in multiband detection. Estimating the threshold value is performed by using the functions of the first and second statistics of the 
received signal. In [21], the Wigner-Ville distribution is used to improve detection performance at a low SNR. In this case, a better decision threshold is defined by reducing the effects of the cross-correlation terms. In [22], using Gauss-Hettite integration, analytical expressions of detection and mean field probabilities on compound Nakagami- $m$ and log-normal fading channels were obtained, and detection performance was investigated. In addition, an optimized threshold value expression was obtained to increase the spectrum detection performance. In [23], an energy detector, using an adaptive dual threshold, is proposed for solving the detection problem. In [24], the authors proposed an adaptive threshold detection algorithm based on an image binarization technique. Here, the dynamic threshold value is estimated based on previous repetition decision statistics, parameters such as SNR, number of instances, and detection probabilities. In [25], a dynamic threshold detection scheme was proposed depending on the noise level present in the received signal. For the measurement of the noise level, a blind technique based was used on the sample covariance matrix values of the received signal.

The disadvantage of the energy sensing method is that it is dependent on noise power. Little change in power of noise can result in large decline in the performance of energy detector due to SNR thresholds. In order to minimize the negative effects caused by noise uncertainty, cooperative spectrum sensing model is defined in the literature [26], [27]. In [26], the researchers proposed a fuzzy logic-based perception format for collaborative energy detection, based on the new reliability factors for local spectrum sensing. The fuzzy logic process consists of three stages. These are the ordering of blurring, the run-in motor and the clearing phase. The performance of the nodes is compared with the performance of the other nodes to try to make the most accurate predictions. When these processes are performed, the reliability factor is defined by using the SNR, detection differences and threshold values, and the detection performance is measured. In [27], energy detector parameters are optimized for the best detection performance. Simulation studies have been carried out on fading channels in relation to the optimal threshold, number of cognitive radio users and number of antennas.

In recent years, it seems that there is a serious increase in working with artificial intelligence and machine learning algorithms (MSA). In [28], the authors proposed a sensing method based on machine learning for solving the spectrum sensing problem. This method is dependent on signal characteristics and the clustering algorithm that is used for classification. The received signals are classified by using the $k$-means clustering algorithm. Class parameters, eigenvalues and covariance were determined, and the performance of the proposed algorithm was investigated. Using the MSA, it is stated that the error probability decreased and the detection performance increased. In [29], the researchers proposed an online learning algorithm-based energy detection for the solution of the spectrum sensing issue. In the study, the balance between missed detection probability and false alarm probability was observed, and new mathematical expressions were obtained for the threshold value expression. In this paper, the work presented in [29] has been expanded and developed by considering various wireless fading channels. More specifically, spectrum detection error distributions are analyzed and derived from mathematical expressions for decision threshold. A novel analytical expression is used to calculate the probability of detection in different fading channels. We compare the theoretical results of detection probability of AWGN, Rayleigh, Rician, Nakagami- $m$ and Weibull fading channels with the simulation results using an online learning algorithm based spectrum sensing. 
The rest of this paper is organized as follows: Section 2 considers theoretical aspects of energy based spectrum sensing. Optimal thresholds are presented with a sufficient optimality condition in Section 3. Section 4, the optimal threshold value expression is redefined and formulated by using the proposed online learning algorithm. Simulation results are discussed in Section 5 and finally the paper is concluded in Section 6.

\section{Related work}

\subsection{System Model}

Spectrum detection is one of the most important components of cognitive radio networks. Among spectrum sensing techniques, energy detection is the most widely used method since it is low complexity and it does not require prior information about of the primary signals. In the energy detection process, the spectrum occupancy decision is based only on the threshold value obtained depending on the noise. To decision the presence or absence of the primary user, the threshold value is compared to the perceived energy. It aims essentially to decide between two states: primary user signal is absent, denoted by $H_{0}$, or primary user signal is present, denoted by $H_{1}$. The decision of energy detector is the test of the following hypothesis:

$$
\begin{aligned}
& H_{0}: Y(n)=W(n), \\
& \quad \text { Primary user absent } \\
& H_{1}: Y(n)=S(n)+W(n), \quad: \text { Primary user present }
\end{aligned}
$$

where $Y(n)$ is the signal received by secondary user and $S(n)$ is primary user's transmitted signal, $W(n)$ is the Additive White Gaussian Noise (AWGN) with zero mean. Fig. 1 shows the basic block diagram of the energy detection.

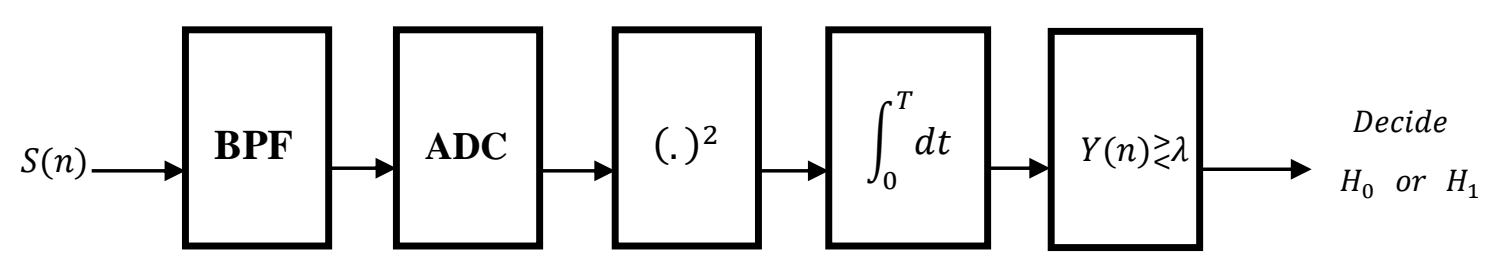

Fig. 1 Energy detector block diagram

In order to estimate the energy of received signal is first pre-filtered by an ideal bandpass filter which has bandwith $W$. The filter output is then squared and integrated over a predefined time interval. The resultant signal is used to formulate a test statistic. The test statistic $T$ compared against a decision threshold value $\lambda$ so as determine spectrum occupancy. The test statistic can be formulated as shown in equation 2 .

T

$=\sum_{n=0}^{N}|Y(n)|^{2}$ 
where $n=0,1,2,3, \ldots \ldots, N$, which represents the number of samples (detection period). If $N$ sample numbers are sufficient, the $T$ statistic distribution, according to the central limit theorem, is Gaussian distribution [30]. The binary hypothesis test is redefined as follows,

$$
H_{0}: \quad T \sim \operatorname{Normal}\left(N \sigma_{n}^{2}+N 2 \sigma_{s}^{4}\right)
$$

$H_{1}: \quad T \sim \operatorname{Normal}\left(\left(\sigma_{n}^{2}\right.\right.$

$\left.+\sigma_{s}^{2}\right), 2 N\left(\sigma_{n}^{2}\right.$

$\left.\left.+\sigma_{s}^{2}\right)^{2}\right)$

where ${\sigma_{n}}^{2}$ and ${\sigma_{s}}^{2}$ are the noise variance and signal variance, respectively.

Test statistic is greater than threshold, $(T \geq \lambda)$, it can be concluded that the primary user is present and hypothesis $H_{1}$ will be declared. Contrary to that, if the test statistic is less than threshold, $(T<\lambda)$, it can be concluded that the primary user is absent and hypothesis $H_{0}$ will be declared. According to [31], the probabilities of detection $P_{d}$ and false alarm $P_{f a}$ are given by,

$P_{d}=P\left(T>\lambda / H_{1}\right)$

$=Q\left(\frac{\lambda-N\left(\sigma_{n}^{2}+\sigma_{s}^{2}\right)}{\sqrt{2 N\left(\sigma_{n}^{2}+\sigma_{s}^{2}\right)^{2}}}\right)$

$P_{f a}=P\left(T>\lambda / H_{0}\right)$

$=Q\left(\frac{\lambda-N \sigma_{n}^{2}}{\sqrt{2 N \sigma_{n}^{4}}}\right)$

where Q-function $Q(x)$ is expressed as follows.

$Q(x)$

$=\frac{1}{\sqrt{2 \pi}} \int_{x}^{\infty} \exp \left(-\frac{y^{2}}{2}\right) d y$

\subsection{Threshold Detection}

The performance of energy sensing-based medots is largely dependent on the previously defined threshold value expression [32], [33]. A threshold is required to decide whether the target signal is absent or present. This threshold determines all spectrum sensing performance metrics. The sensing performance of the energy detector is measured according to two metrics. The performance metrics $P_{d}$ and $P_{f a}$ over AWGN channels will then be given as [34], [35]:

$P_{d}$

$=\frac{1}{2} \operatorname{erfc} \frac{\lambda-\mu_{1}}{\sqrt{2} \sigma_{1}}$

$P_{f a}$

$=\frac{1}{2} \operatorname{erfc} \frac{\lambda-\mu_{0}}{\sqrt{2} \sigma_{0}}$ 
where erfc is the complementary error function. It then follows that the mean and the variance of the test statistic could be represented as shown below in Equation 10 to 13.

$$
\begin{aligned}
& \mu_{0}=N \sigma_{n} \\
& \mu_{1}=N \sigma_{n}^{2}(\gamma+1)^{2} \\
& \sigma_{0}^{2}=2 N \sigma_{n}^{4} \\
& \sigma_{1}^{2}=2 N \sigma_{n}^{4}(\gamma+1)^{2}
\end{aligned}
$$

Probability of miss detection would be given as,

$P_{m}$

$=1$

$-P_{d}$

The balance between $P_{f a}$ and $P_{m}$ should be considered when determining the threshold value for the energy detector. $P_{d}$ should be maximized, while $P_{f a}$ should be minimized. This is called the constant false alarm rate (CFAR) detection scheme. $P_{m}$ can be set to a minimum value, or $P_{f a}$ can be reduced to a minimum by fixing $P_{d}$ to a maximum value. In practice, the threshold is normally chosen so as to meet a certain $P_{f a}$, in situations where only the noise power needs to be known. Depending on the balance between $P_{d}$ and $P_{f a}, \lambda$ for a certain $P_{f a}$ value is derived as:

$\lambda$

$=Q^{-1}\left(P_{f a}\right) \sqrt{2 N}$

$+(N) \sigma_{n}$

Due to this threshold at low SNR, the detection performance is greatly reduced. What is important here is to improve the low SNR perception performance. For this reason, the optimal threshold value is defined by using the total error probability, $P_{e}$, which is dependent on $P_{f a}$ and $P_{m}$. If a priori information of the spectrum occupancy is available and given by $P H_{1}, P H_{0}$, which represent the probabilities of primary user presence and absence respectively, where $P H_{1}+P H_{0}=1$. The total error probability is the sum of $P_{f a}$ and $P_{m}$ weights. $P_{e}$ can be given as

$P_{e}$

$=P H_{0} P_{f a}$

$+\mathrm{PH}_{1} P_{m}$

the minimization problem can be represented as 
$\lambda$

$=\operatorname{argmin}_{\lambda}\left(P H_{0} P_{f a}\right.$

$\left.+\mathrm{PH}_{1} P_{m}\right)$

the threshold can be obtained by satisfying following conditions given below [35].

$\frac{\partial P_{f a}}{\partial \lambda}+\frac{\partial P_{m}}{\partial \lambda}$

$=0$

$\frac{\partial^{2} P_{e}}{\partial \lambda^{2}}$

$<0$

From the equations $(8,9)$ on differentiating $P_{f a}$ and $P_{m}$ are given as follows:

$\frac{\partial P_{f a}}{\partial \lambda}$

$=-\frac{1}{\sqrt{2 \pi} \sigma_{0}} e^{-\left(\frac{\left(\lambda-\mu_{0}\right)^{2}}{\sqrt{2} \sigma_{0}}\right)}$

$\frac{\partial P_{m}}{\partial \lambda}$

$=-\frac{1}{\sqrt{2 \pi} \sigma_{1}} e^{-\left(\frac{\left(\lambda-\mu_{1}\right)^{2}}{\sqrt{2} \sigma_{1}}\right)}$

Using the equations $17,18,19$ and 20 the threshold value is redefined as follows.

$\lambda$

$=\frac{-b+\sqrt{b-a c}}{a}$

where

$a$

$=\sigma_{1}^{2}$

$-\sigma_{0}^{2}$

$b$

$=\sigma_{0}^{2} \mu_{1}$

$-\sigma_{1}^{2} \mu_{0}$ 
$=\sigma_{1}^{2} \mu_{0}-\sigma_{0}^{2} \mu_{1}$

$-\frac{\sigma_{1}^{2} \sigma_{0}^{2}}{\ln \left(\frac{\sigma_{1}}{\sigma_{0}}\right)}$

\section{Proposed Adaptive Threshold Optimization}

In cognitive radio systems, the detection performance of the energy detector depends on the threshold value selection. When developing spectrum detection models, it is aimed that the noise and primary user signals are fully distinguished. Developed models are generally evaluated based on parameters such as accuracy and correct positive rate. However, the actual performance can be analyzed by using backwardly artificially generated estimates in the measurements. The performance of the energy detection method, which is used to solve the spectrum sensing problem, is dependent on the threshold value that is defined according to the noise power.

The fundamental nature of spectrum sensing is a binary hypothesis-testing problem that is dependent on the threshold value expression. This relationship is illustrated in Figure 2. This shows the expected distribution of a difference between two groups under $H_{0}$ and $H_{1}$. It should be clear that, if we increase the type I error rate (false positive (FP) or false alarm), we reduce the type II error rate (false negative (FN) or missed detection), and vice versa. This results in a change in the accuracy of the $H_{0}$ (true negative (TN)) and $H_{1}$ (true positive (TP)) hypotheses, depending on the change in total error probability. In this study, the relationship between the probability of false alarm and the probability of missed-detection is investigated by using an online learning algorithm. Two classes are constructed by classifying the negative and positive data, as shown in Fig. 2. Type I and II error parameters and correct perception parameters are analyzed. Critical thresholds are determined for these classes, creating a gray area.

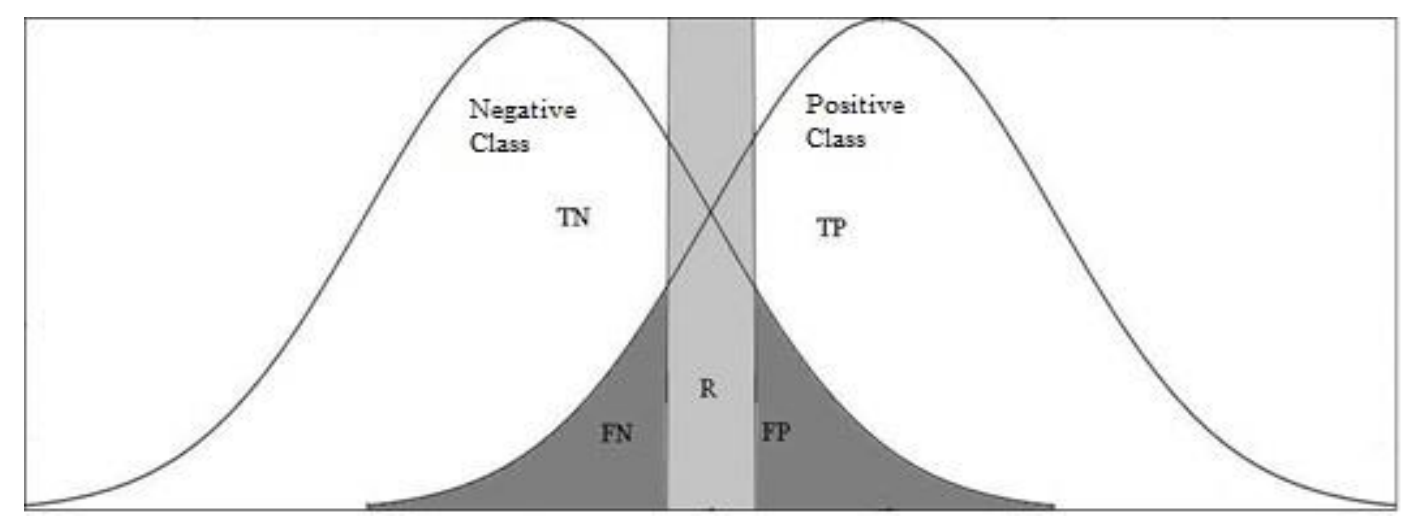

Fig. 2 Statistical Distribution Curves Related to Classes

The process steps for obtaining the optimal threshold value with the aid of the online learning algorithm are given below.

Stage 1: Data collection and Pre-processing 
The gauss distribution curves of $H_{1}$ (signal present) and $H_{0}$ (signal absent) are obtained by using the threshold value expression in Equation 22. Two classes are constructed by classifying the negative and positive data, as shown in Fig. 2. Type I and II error parameters and correct perception parameters are analyzed. Critical thresholds are determined for these classes, creating a gray area.

i. Each $\left(N_{i}, P_{i}\right)$ values are determined and classes are created.

ii. Critical thresholds value expressions of the two classes are defined $\left(\lambda_{N}, \lambda_{p}\right)$.

The data in the gray area, defined as $\mathrm{R}$ in Figure 2, is divided into subclasses using the $k$-means algorithm $(k=4)$. Generated classes are graded in terms of performance levels, taking into account the type I and II errors. In order to increase the success levels of the successful classes, error analysis and error coefficients and weight sequences were obtained. An improvement is defined with the aid of weight sequences. iii. Subclasses are created within the remaining gray area between two threshold values $\left(X_{1,2,}, \ldots \ldots \ldots, X_{n}\right)$.

\section{Stage 2. Computation on the data set}

The data obtained during the data collection and pre-processing phase are defined as weight and error statements to be used in the model. These expressions respectively;

i. Weights are defined for each subclass. $\left(w_{t}\right)$

ii. Averages of weights are found. It is expressed as show in equation 25;

$w_{t}$

$=\frac{w_{t, i}}{\sum_{i=0}^{N} w_{t, i}}$

iii. The data are classified and the total error rate is obtained. It could be represented as shown below in equation 26 and equation 27.

$$
\begin{aligned}
& E_{T}=\varepsilon_{t}= \\
& \min \sum_{i=0}^{N} w_{t} c_{i} \\
& c_{i}= \\
& \left\{\begin{array}{c}
0, h_{t}\left(H_{i}, X_{i}, Y_{i}, P_{i}\right)=y_{i} \\
1, h_{t}\left(H_{i}, X_{i}, Y_{i}, P_{i}\right) \neq y_{i}
\end{array}\right.
\end{aligned}
$$

iv. Incorrect Positive Error ( $\mathrm{H}_{1} /$ Incorrect Detection) is expressed in equation 28 as follows;

$$
\begin{aligned}
& E_{F P} \\
& =\sum_{i=0}^{p} w_{t, i} c_{i}
\end{aligned}
$$

v. Incorrect Negative Error $\left(\mathrm{H}_{0} /\right.$ Incorrect Detection $)$ is expressed in 29. 
$E_{F N}$

$=\sum_{i=0}^{N} w_{t, i} c_{i}$

vi. Classification probabilities and ratios in order are as follows equation 30-33.

$P_{F P}$

$=\frac{E_{F P}}{E_{T}}$

$P_{F N}$

$=\frac{E_{F N}}{E_{T}}$

$T P R$

$=\frac{T P}{T P+T N}$

TNR

$=\frac{T N}{T N+F P}$

vii. Mathews Correlation Coefficient can be represented shown in equation 34 .

MCC

$=\frac{T P * T N-F P * F N}{\sqrt{(T P+F P)(T P+F N)(T N+F P)(T N+F N)}}$

viii. Improvement coefficient $\left(p_{i}\right)$ can be formulated by equation 35 below;

$p_{i}$

$=\left[\log \left(\frac{1-\varepsilon_{t}}{\varepsilon_{t}}\right)\right]\left(\frac{1-P_{F N}}{P_{F P}}\right)$

* MCC

\section{Stage 3: Training Phase}

We are provided with a training data set $\left(X_{i}, Y_{i}\right), i=1,2,3, \ldots \ldots, N$ where $X_{i}$ represents an $n$-dimensional continuous valued vector and $Y_{i}\{0,1\}$ represents the corresponding class label with " 0 " for normal and " 1 " for anomaly. The proposed method has two steps: 1) training and 2) testing. During training, the $k$-means-based anomaly detection method are first applied to partition the training space into $\mathrm{k}$ disjoint clusters $C_{1}, C_{2}, C_{3}, \ldots \ldots, C_{N}$. Then, decision tree is trained with the instances in each $k$-means cluster. The $k$-means method ensures that each training instance is associated with only one cluster. However, if there are any subgroups or overlaps within a cluster, the decision tree trained on that cluster refines the decision boundaries by partitioning the instances with a set of if then rules over the feature space. In the testing phase, we have two 
subdivided phases 1) Selection Phase and 2) Classification Phase. In selection phase, compute the Euclidean distance for every testing instance and find the closest cluster. Compute the decision tree for the closest cluster. In classification phase, the data are separated according to the detection successes. Finally in this phase, threshold will learn from the best learner in class. Learner modification is expressed as,

$$
\begin{aligned}
& \lambda_{i} \\
& =\lambda \\
& +p_{i}\left[\frac{\min \left(\text { energy }_{i}\right)+\max \left(\operatorname{energy}_{i}\right)}{2}\right]
\end{aligned}
$$

\section{Stage 4: Learner Phase}

In this phase, through comparing the advantages and disadvantages between other two learners, the learners $\lambda_{i}^{\text {new }}$ will learn from their advantages which draw on the idea of differential evolution algorithm. Randomly select two learners $\lambda_{i}$ and $\lambda_{j}$, where $i \neq j$. Learner modification is expressed as

$$
\begin{gathered}
\lambda_{i}^{\text {new }}=\lambda_{i}^{\text {old }}+\operatorname{rand}() *\left(\lambda_{i}-\lambda_{j}\right) \quad \text { if } P_{i} \\
>P_{j} \\
\lambda_{i}^{\text {new }}=\lambda_{i}^{\text {old }}+\operatorname{rand}() *\left(\lambda_{j}-\lambda_{i}\right) \quad \text { if } P_{i} \\
<P_{j}
\end{gathered}
$$

where $\operatorname{rand}(\mathrm{)})$ is a uniformly distributed random number between " 0 " and " 1 ". Accept $\lambda_{i}^{\text {new }}$ if it gives a optimum threshold.

\section{Simulation Results}

In this section, numerical results are presented to verify the effectiveness of our proposed algorithms. The performance of the energy detector may be characterized by using the receiver operating characteristic (ROC) curve in cognitive radio networks. ROC curves are generated by plotting either detection probability versus false alarm probability or missed detection probability versus false alarm probability Detection probability and false alarm probability depend on the threshold, number of samples, fading parameters, number of diversity branches, and average SNR. In fig.3 - fig.7, simulation results are provided to compare our (online learning algorithm) threshold selection with a conventional (dynamic) threshold selection (calculated from $P_{f a}=0.1$ ). ROC curves are plotted for different SNR values.

Fig. 3 shows the ROC curve for AWGN channel. As can be seen, the performances of the proposed algorithm for different SNR scenarios are higher than those of conventional algorithms. Dynamic Threshold (-5dB): $P_{d}=0.6860$. Online Learning Threshold (-5dB): $P_{d}=0.6915$. Dynamic Threshold (-10dB): $P_{d}=0.3347$. Online Learning Threshold (-10dB): $P_{d}=0.3439$. Fig. 4 -fig.7 illustrate the ROC curves for Rayleigh, Nakagami- $m$, Rician and Weibull channels, respectively. When the graphs are examined, it is clearly seen that the detection performance of cognitive radio increases with the proposed method. In addition, detection probability is less in Rayleigh fading channel when compared to AWGN channel and other fading channels. This situation is clearly 
seen in fig. 4 and table 1. In fig.6, we can see that the performance of energy detector in Rician fading channel is better than in the other channels (Rician factor $\mathrm{K}=5$ ).

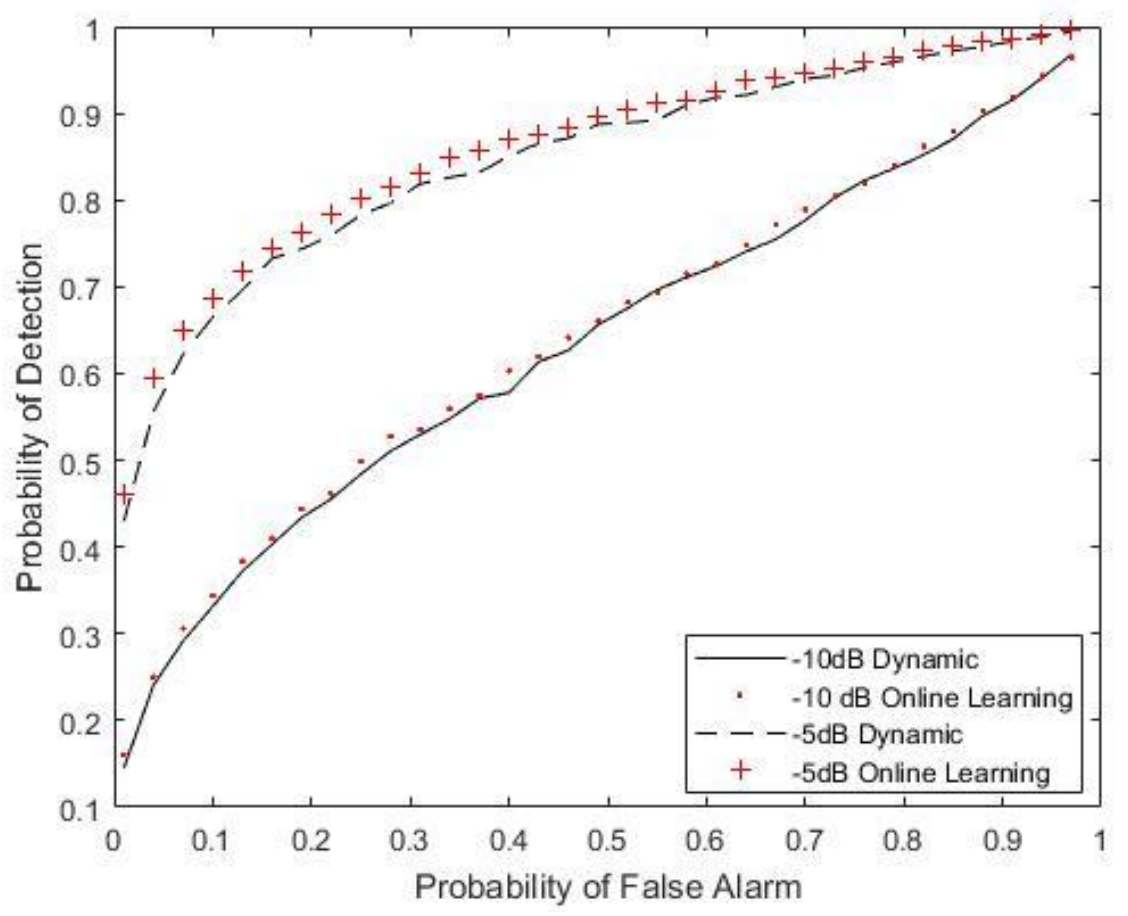

Fig. 3 The ROC curve over the AWGN channel for different SNRs

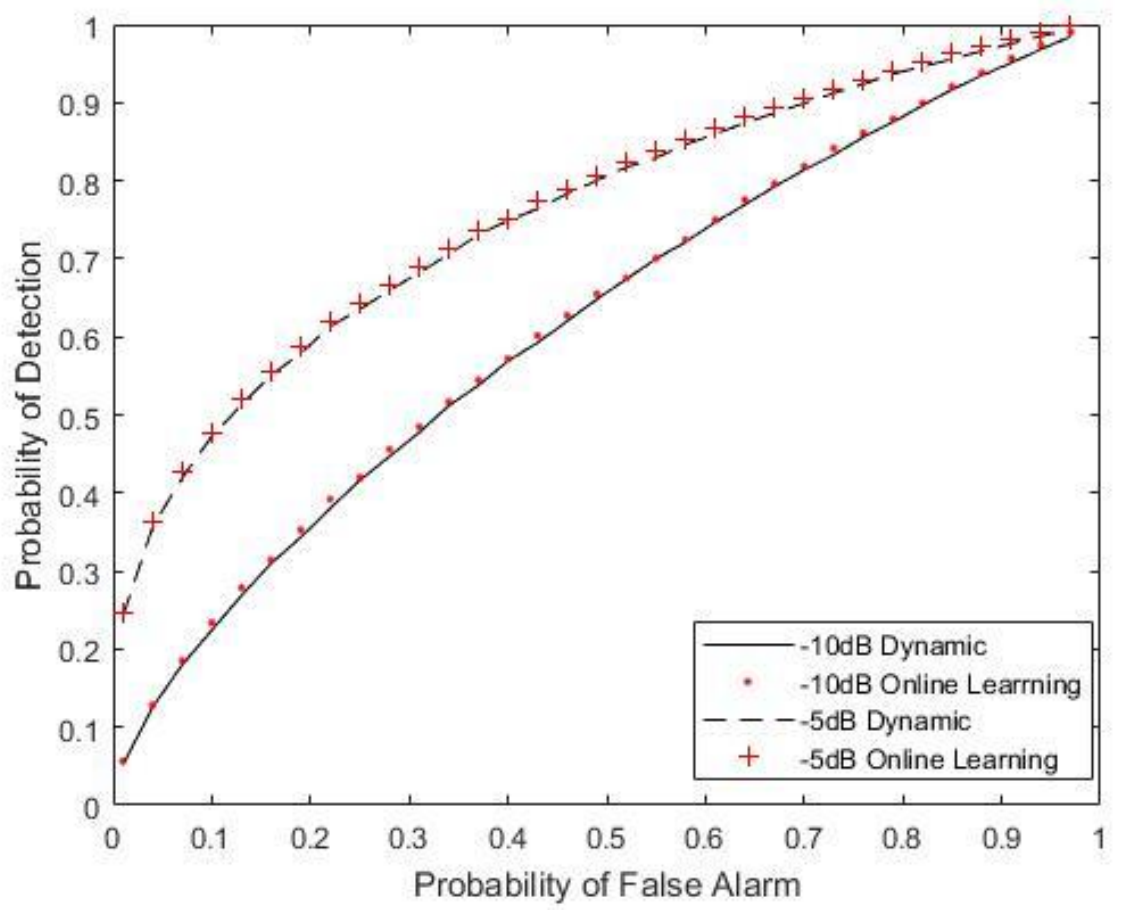

Fig. 4 The ROC curve over the Rayleigh channel for different SNRs 


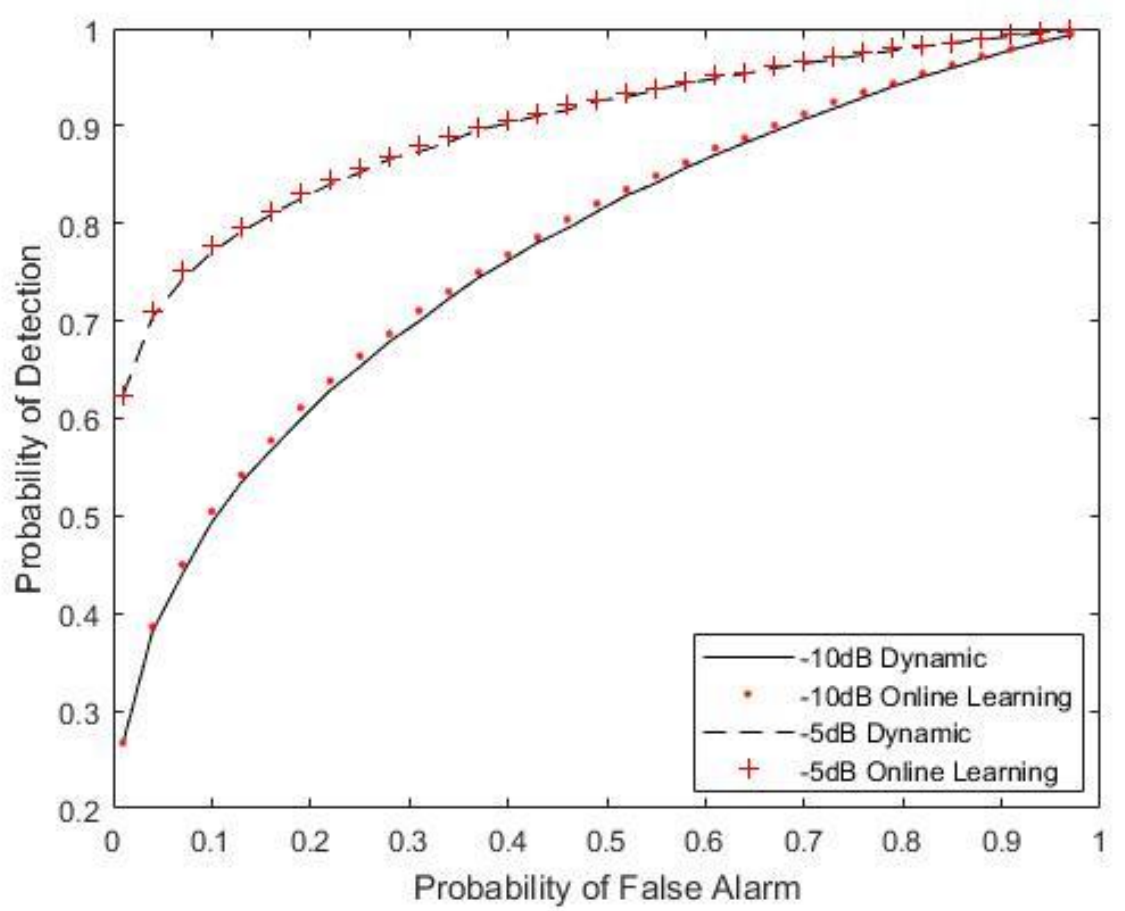

Fig. 5 The ROC curve over the Nakagami- $m$ channel for different SNRs and $m=3$

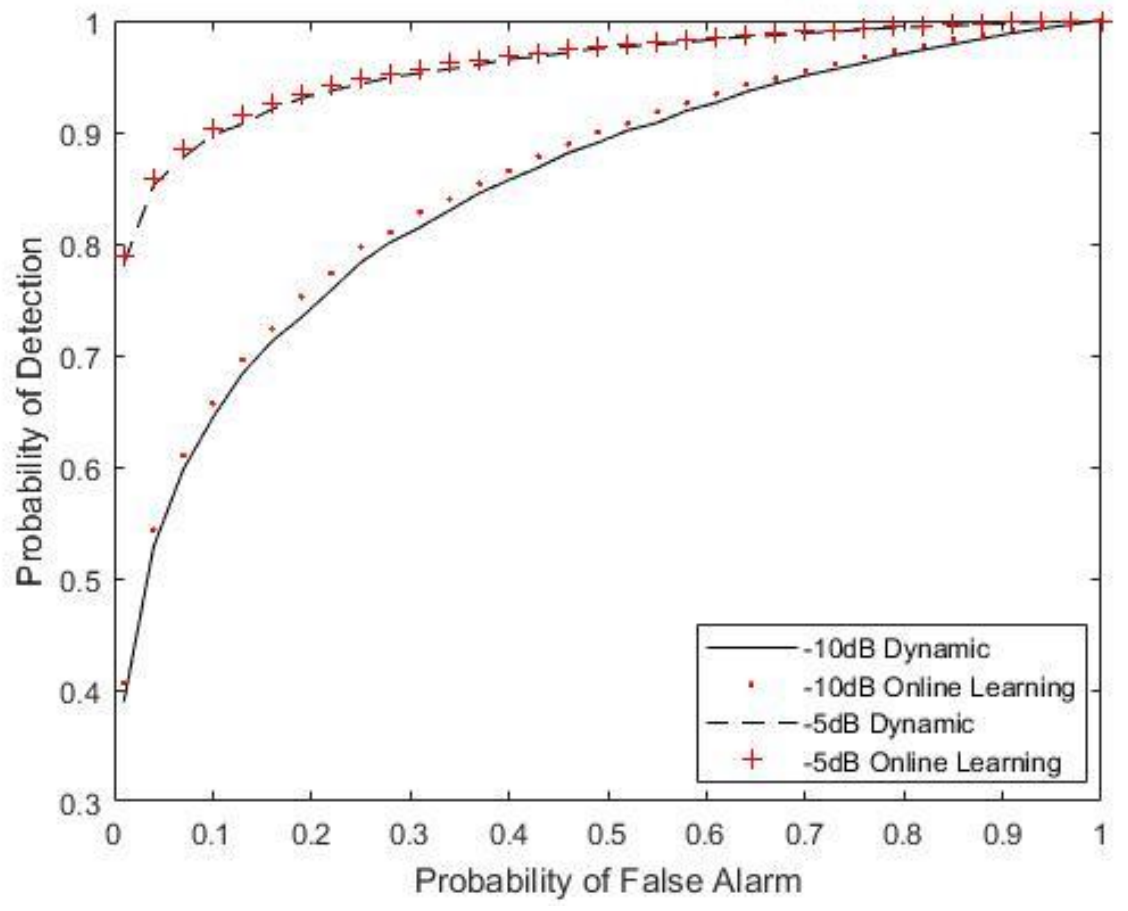

Fig. 6 The ROC curve over the Rician channel for different SNRs and $\mathrm{K}=5$ 


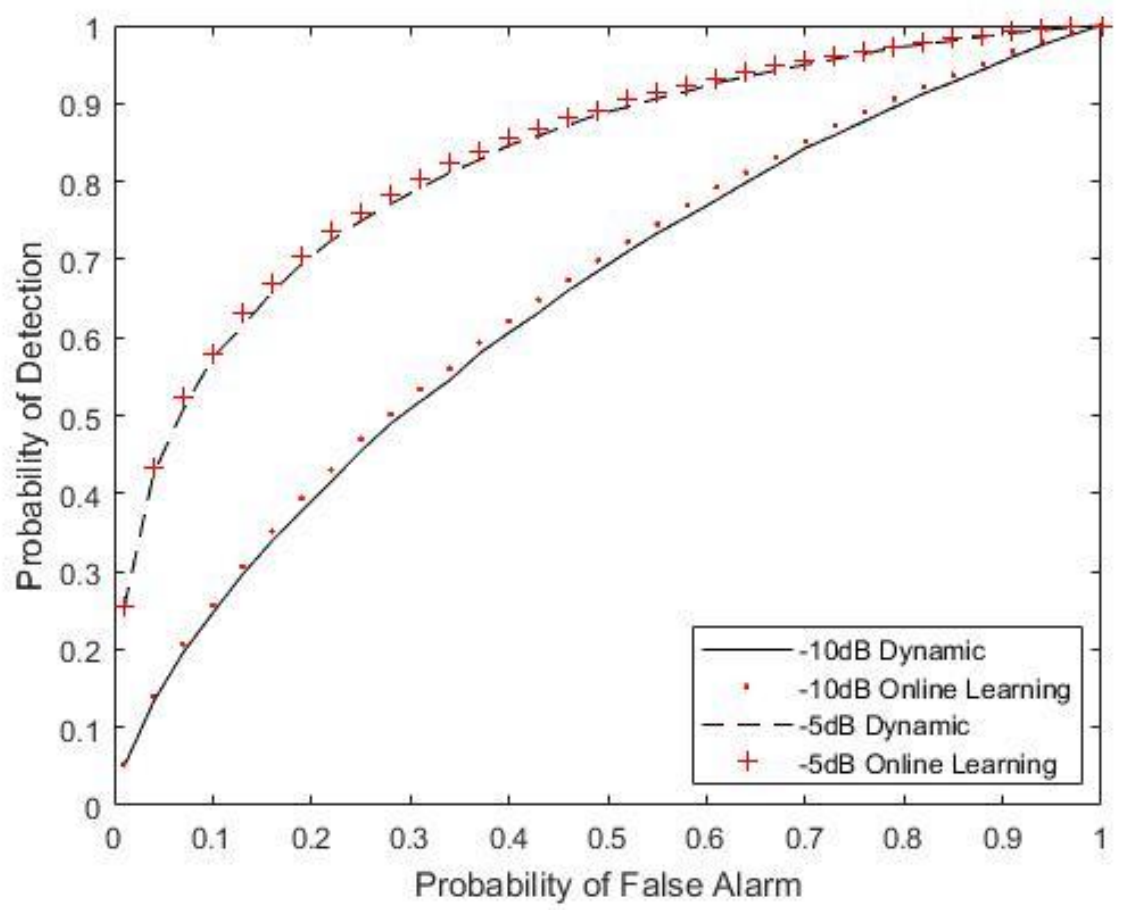

Fig. 7 The ROC curve over the Weibull channel for different SNRs and $a=3$

Fig.7 shows that, for energy detection in Weibull fading channels, ROC curves move to the upper left corner with the proposed method, confirming better overall detection performance.

The threshold and detection probabilities for different fading channels are given in table 1. It is found that for $P_{f a}=0.1$, there is improvement in detection probability in the spectrum sensing, in the complete low SNR region, which is of great significance. According to the table, detection probability is the highest in Rician fading channel when compared to Rayleigh, Nakagami-m, Weibull fading channels and AWGN channel.

Table 1 Threshold value and probability of detection for AWGN and fading channels.

\begin{tabular}{|c|c|c|c|c|}
\hline Channel & SNR & Threshold & & $\begin{array}{l}\text { Probability of } \\
\text { Detection }\left(\boldsymbol{P}_{\boldsymbol{d}}\right)\end{array}$ \\
\hline \multirow{4}{*}{ AWGN } & \multirow[b]{2}{*}{$-5 \mathrm{~dB}$} & Dynamic Threshold Value $\left(\lambda_{d}\right)$ & $6^{11.812}$ & 0.6860 \\
\hline & & Online Learning Threshold Value $\left(\lambda_{\text {new }}\right)$ & $2^{11.773}$ & 0.6915 \\
\hline & \multirow[b]{2}{*}{$-10 \mathrm{~dB}$} & Dynamic Threshold Value $\left(\lambda_{d}\right)$ & $7^{11.801}$ & 0.3347 \\
\hline & & Online Learning Threshold Value $\left(\lambda_{\text {new }}\right)$ & $3^{11.684}$ & 0.3439 \\
\hline \multirow{4}{*}{ Rayleigh } & \multirow[b]{2}{*}{$-5 \mathrm{~dB}$} & Dynamic Threshold Value $\left(\lambda_{d}\right)$ & $4^{13.155}$ & 0.4741 \\
\hline & & Online Learning Threshold Value $\left(\lambda_{\text {new }}\right)$ & $8^{12.832}$ & 0.4791 \\
\hline & \multirow[b]{2}{*}{$-10 \mathrm{~dB}$} & Dynamic Threshold Value $\left(\lambda_{\mathrm{d}}\right)$ & $0^{13.151}$ & 0.2316 \\
\hline & & Online Learning Threshold Value $\left(\lambda_{\text {new }}\right)$ & $0^{12.882}$ & 0.2352 \\
\hline Nakagami- $m$ & & Dynamic Threshold Value $\left(\lambda_{d}\right)$ & 18.045 & 0.8636 \\
\hline
\end{tabular}


$-5 \mathrm{~dB}$

Rician

Weibull

$-5 \mathrm{~dB}$

$\begin{array}{ll} & \text { Online Learning Threshold Value }\left(\lambda_{\text {new }}\right) \\ -10 \mathrm{~dB} & \text { Dynamic Threshold Value }\left(\lambda_{\mathrm{d}}\right) \\ & \text { Online Learning Threshold Value }\left(\lambda_{\text {new }}\right) \\ -5 \mathrm{~dB} & \text { Dynamic Threshold Value }\left(\lambda_{\mathrm{d}}\right)\end{array}$

Online Learning Threshold Value $\left(\lambda_{\text {new }}\right)$

Dynamic Threshold Value $\left(\lambda_{\mathrm{d}}\right)$

$-10 \mathrm{~dB}$

Online Learning Threshold Value $\left(\lambda_{\text {new }}\right)$

Dynamic Threshold Value $\left(\lambda_{\mathrm{d}}\right)$

Online Learning Threshold Value $\left(\lambda_{\text {new }}\right)$

Dynamic Threshold Value $\left(\lambda_{\mathrm{d}}\right)$
7

16.701

3

18.075

9

16.639

3

12.102

2

11.847

2

12.045

7

11.848

8

12.064

4

11.890

7

12.058
$11.863 \quad 0.5028$

0.8689

0.6772

0.6895

0.9492

0.9530

0.8015

0.8104

0.7716

0.7821

0.4853

It has been observed that the online learning algorithm has extremely good performance compared to other traditional methods. This is because conventional methods offer a solid threshold value model. The proposed method in this study has made the threshold value expression flexible. Furthermore, with the proposed online learning algorithm, the spectrum detection performance has been made more sensitive to the changes in the communication channels.

\section{Conclusions}

In this paper, we have analyzed the performance of energy detection in low SNR, deriving new threshold expression. In the first instance, the new threshold value expression was formulated using the online learning algorithm to minimize the total error rate due to limited false alarm and miss-detection probabilities. Subsequently, the detection performance is analyzed theoretically over AWGN and different fading channels (Rayleigh, Nakagami-m, Rician, Weibull). The simulation results show that a significant improvement in spectrum detection efficiency compared to conventional detection schemes.

\section{Declarations}

\section{Abbreviations}

SNR: Signal-to-noise ratio

FCC: Federal communications commission

MSA: Machine learning algorithms 
AWGN: Additive White Gaussian Noise

ROC: Receiver operating characteristic

CFAR: Constant false alarm rate

FP: False positive

FN: False negative

TN: True negative

TP: True positive

\section{Ethics approval and consent to participate}

This article does not contain any studies with human participants or animals performed by any of the authors.

\section{Consent for publication}

All authors agree to submit this version and claim that no part of this manuscript has been published or submitted elsewhere.

\section{Availability of data and materials}

The datasets used and/or analysed during the current study are available from the corresponding author on reasonable request.

\section{Competing interests}

The authors declare that they have no competing interests.

\section{Funding}

This study is supported by Erciyes University Scientific Research Projects Coordination Unit. (Project Number: FDK-2016-6908)

\section{Authors Contributions}

K.K. has modeled and executed the research. İ.D. have supervised the research. K.K. performed the system simulations. K.K. and İ.D. revised the equations and contributed to the writing of the manuscript. İ.D. has enhanced the quality of the research by their valuable comments and suggestions in data analysis and discussion. The authors read and approved the final manuscript.

\section{Acknowledgements}

This study is supported by Erciyes University Scientific Research Projects Coordination Unit. (Project Number: FDK-2016-6908) 


\section{Author information}

Department of Divriği Nuri Demiră̆ Vocational High School, Sivas Cumhuriyet University in Sivas, 58300 Divriği, Sivas, Turkey

Kenan Koçkaya

Department of Electronic Engineering, Erciyes University in Kayseri, 38280 Talas Kayseri, Turkey İbrahim Develi

\section{References}

1. FCC, Federal Communications Commission Spectrum Policy Task Force, Report of the Spectrum Efficiency Working Group, (Technical Report). (2002). USA.

2. Tuna, E. and Karagöz, M, A New Approach in Spectrum Allocation for Next Generation Networks: Cognitive Radio, International Journal of Engineering Research and Development,4(1),25-32(2012) https://dergipark.org.tr/tr/pub/umagd/issue/31723/345859

3. Mitola, J. and Maguire, G. Q, Cognitive Radio: Making Software Radios More Personal, IEEE Personal Communications Magazine, 6(4), 13-18 (1999) DOI: $\underline{10.1109 / 98.788210}$

4. Mitola, J, Cognitive Radio: An Integrated Agent Architecture for Software Defined Radio, Ph. D. Dissertation, KTH Royal Institute of Technology, 2000. Stockholm, Sweden.

5. Atapattu, S, Energy Detection Based Cooperative Spectrum Sensing in Cognitive Radio Networks, IEEE $\begin{array}{llll}\text { Transactions on } \quad \text { Wireless } \quad \text { Communications, } & \text { 10(4), }\end{array}$

DOI: $\underline{\text { 10.1109/TWC.2011.012411.100611 }}$

6. Zeng Y., Koh C. L., and Liang Y.-C, Maximum Eigenvalue Detection: Theory and Application, IEEE International Conference on Communications (ICC '08), 4160-4164 (2008) DOI: $\underline{\text { 10.1109/TCOMM.2009.06.070402 }}$

7. Akyildiz, I. F., Brandon, F. Lo and Ravikumar, B, Cooperative Spectrum Sensing in Cognitive Radio Networks: A survey, Physical Communication, 4(1), 40-62, (2011) https://doi.org/10.1016/j.phycom.2010.12.003

8. Liangping Ma, Yingxue Li and Demir. A, Matched Filtering Assisted Energy Detection for Sensing Weak Primary User Signals, IEEE International Conference on Acoustics, Speech and Signal Processing(ICASSP), 3149 - 3152 (2012)

DOI: $\underline{10.1109 / \text { ICASSP.2012.6288583 }}$

9. Bektaş, C. and Akan, A, Wavelet Transformation Approach in Energy Based Spectrum Sensing, XX. Signal Processing and Communications Applications Conference, 18-20 Nisan, Muğla, 1-4, (2012) DOI: $\underline{10.1109 / S I U .2012 .6204424}$

10. Ma J., Li G., and Juang B.-H, Signal Processing in Cognitive Radio, Proceedings of the IEEE, 97(5), 805-823. (2009) DOI: $10.1109 /$ JPROC.2009.2015707 
11. Chen H.-S., Gao W., and Daut D. G, Signature Based Spectrum Sensing Algorithms for IEEE 802.22 WRAN, In Proceedings of the IEEE International Conference on Communications (ICC '07), 6487-6492 (2007) DOI: $\underline{\text { 10.1109/ICC.2007.1073 }}$

12. Haykin, S, Cognitive Radio: Brain Empowered Wireless Communications, IEEE Journal on Selected Areas in Communications, 23(2), 201-220 (2005)

DOI: $10.1109 / \mathrm{JSAC} .2004 .839380$

13. Gardner W. A, Exploitation of Spectral Redundancy in Cyclostationary Signals, IEEE Signal Processing Magazine, 8(2), 14-36 (1991) DOI: $10.1109 / 79.81007$

14. Tsinos C. and Berberidis K, Decentralized Adaptive Eigenvalue-Based Spectrum Sensing for Multiantenna Cognitive Radio Systems, IEEE Transactions on Wireless Communications, 14(3), 1703-1715 (2015) DOI: 10.1109/TWC.2014.2372756.

15.Zeng, Y., Liang Y-C, Eigenvalue-Based Spectrum Sensing Algorithms for Cognitive Radio, IEEE Transactions Communications, 57(6), 1784-1793 (2009)

DOI: $10.1109 / \mathrm{TCOMM} .2009 .06 .070402$

16. Tsinos C. and Berberidis K, Adaptive Eigenvalue-Based Spectrum Sensing For Multi-Antenna Cognitive Radio Systems, Proc. of the 2013 IEEE Int. Conference on Acoustics, Speech and Signal Processing, ICASSP-2013, 4454-4458 (2013) DOI: 10.1109/ICASSP.2013.6638502

17. Shakir, M. Z., Rao, A. and Alouini M.S, Generalized Mean Metector for Collaborative Spectrum Sensing, IEEE Transactions on Communications, 61(4), 1242-1253 (2013) DOI: $\underline{\text { 10.1109/TCOMM.2013.13.110594 }}$

18. Yücek, T. and Arslan, H, A Survey of Spectrum Sensing Algorithms for Cognitive Radio Applications, IEEE Communications Surveys \& Tutorials, 11(1), 116-130 (2009)

DOI: $10.1109 /$ SURV.2009.090109

19. Sharma, S., Bogale, T., Chatzinotas, S., Ottersten, B., Le., L. and Wang, X, Cognitive Radio Techniques under Practical Imperfections: A Survey, IEEE Communications Surveys \& Tutorial, 17(4), 1858-1884 (2015) DOI: $10.1109 /$ COMST.2015.2452414

20. Gorcin, A. Qaraqe K.A., Celebi H., Arslan H, An Adaptive Threshold Method for Spectrum Sensing in Multi-channel Cognitive Radio Networks, 17th International Conference on Telecommunications (ICT'10), $425-429$ (2010)

\section{DOI: $\underline{10.1109 / \text { ICTEL.2010.5478783 }}$}

21. Ajadi, W. O., Sani, S. M. and Tekanyi, A. M. S, Estimation of an Improved Spectrum Sensing Threshold for Cognitive Radio using Smoothed Pseudo Wigner-Ville Distribution, International Journal of Computer Applications (0975 - 8887), 168(12), 30-33 (2017) DOI: $\underline{10.5120 / i j c a 2017914503}$

22. Pappu K. V., Sanjay K. S. and Priyanka J, Performance Evolution of ED-Based Spectrum Sensing in CR over Nakagami- $m /$ Shadowed Fading channel with MRC Reception, International Journal of Electronics and Communications (AUE), 83, 512-518 (2018)

DOI: $10.1016 /$ j.aeue.2017.11.005 
23. Bagwari, A. and Tomar, G. S, Adaptive Double-Threshold Based Energy Detector for Spectrum Sensing in Cognitive Radio Networks, International Journal of Electronics Letters, (1), 24-32 (2013) DOI: $\underline{10.1080 / 21681724.2013 .773849}$

24. Muralidharan, A., Venkateswaran, P., Ajay, S. G. D., Prakash, A., Arora, M. and Kirthiga, S, An Adaptive Threshold Method for Energy Based Spectrum Sensing in Cognitive Radio Networks, International Conference on Control, Instrumentation, Communication and Computational Technologies, 8-11 (2015) DOI: $10.1109 /$ ICCICCT.2015.7475239

25. Arjoune, Y., Mrabet, E. Z., Ghazi, E. H. and Tamtaoui, A, Spectrum Sensing: Enhanced Energy Detection Technique Based on Noise Measurement, 2018 IEEE 8th Annual Computing and Communication Workshop and Conference (CCWC), 828-834 (2018)

DOI: $\underline{10.1109 / C C W C .2018 .8301619}$

26. Tallataf, R., Adnan, R. and Ahmad N. A, Reliability Factors Based Fuzzy Logic Scheme for Spectrum Sensing. World Academy of Science, Engineering and Technology International Journal of Information and Communication Engineering, 12(2), 84-89 (2018) DOI: 10.1109/ICASET.2018.8376914

27. Ranjeet, M., Nallagonda, S., and Anuradha, S, Optimization Analysis of Improved Energy Detection based Cooperative Spectrum Sensing Network in Nakagami- $m$ and Weibull Fading Channels, Journal of Engineering Science and Technology Review, 10(2), 114-117. (2017) DOI: $10.25103 /$ jestr.102.14

28. Yongwei, Z., Pin, W.,Shunchao, Z., and Yonghua, W, A Spectrum Sensing Method Based on Signal Feature and Clustering Algorithm in Cognitive Wireless Multimedia Sensor Networks, Hindawi Advances in Moltimedia, 1-11 (2017)

DOI: $\underline{10.1155 / 2017 / 2895680}$

29. Koçkaya, K. and Develi, I. Optimum Threshold Model Based on Online Learning Algorithm for Spectrum Detection in Cognitive Radio Networks (In Turkish). ISAS 2018, SETSCI Conference Indexing System, 2 , 434-439 (2018)

30. Urkowitz, H, Energy Detection of Unknown Deterministic Signals, Proceedings of the IEEE, 523-531 (1967) DOI: $\underline{10.1109 / P R O C .1967 .5573}$

31. Pankaj, V. and Brahmjit, S, Threshold Optimization in Energy Detection Scheme for Maximizing the Spectrum Utilization, ELSEVIER, Procedia Computer Science, 93, 191-198, (2016) DOI: $\underline{10.1016 / j . p r o c s .2016 .07 .200}$

32. Xuping, Z. and Jianguo, P, Energy-Detection Based Spectrum Sensing for Cognitive Radio. IET Conference on Wireless, Mobile and Sensor Networks, Shanghai, December 12-14, 944-977 (2007) DOI: 10.1049/cp:20070306

33. Lee, Y.H. and Oh, D.C, Energy Detection Based Spectrum Sensing for Sensing Error Minimization in Cognitive Radio Networks, International Journal of Communication Networks and Information Security (IJCNIS), 1(1), 1-5 (2009) Corpus ID:15029032

34. Kozal, ASB. Merabti, M. and Bouhafs, F, An İmproved Energy Detection Scheme for Cognitive Radio Networks in Low SNR Region, IEEE Symposium on Computers and Communications, 684-689 (2012)

DOI: $10.1109 /$ ISCC.2012.6249377 
35. Raman, D. and Singh, N.P, Improved Threshold Scheme for Energy Detection In Cognitive Radio Under Low SNR, Association of Computer Electronics and Electrical Engineers, 251-256 (2013) DOI: $\underline{\text { 03.AETS.2013.3.122 }}$ 